\title{
Informes
}

\section{La autonomía de las personas con discapacidad visual y los programas de rehabilitación en tiempos de pandemia}

\author{
Independence for people with visual disability \\ and rehabilitation programmes in the midst of a pandemic \\ M. J. Bellón Fernández, C. Blocona Santos, M. Á. Matey García
}

\section{Resumen}

Uno de los retos más comprometidos que tienen que afrontar las personas con discapacidad visual en su día a día es el desplazamiento. La movilidad autónoma y segura requiere no solo el uso de muchas habilidades perceptivas, conceptuales y de orientación, sino también un entorno seguro e intuitivo, cuyas consignas para la toma de decisiones sean fácilmente interpretables. Las medidas sanitarias y de seguridad, implantadas a raíz de la pandemia, han venido a sumar nuevas dificultades a la orientación y movilidad de este colectivo, que se detallan en este artículo. En la época de la supremacía visual, plantear soluciones para estos conflictos no es fácil ni realista, ya que no siempre dependen de las capacidades personales, resultando imprescindible que cualquier regulación y/o cambio en el entorno se adopte bajo el prisma de cómo se percibe la información cuando no hay visión o esta es muy deficiente. En el artículo se reflejan también los cambios que se han producido en la prestación del Servicio de Rehabilitación de la ONCE en tiempos de pandemia.

\section{Palabras clave}

Desplazamiento autónomo. Distancia de seguridad. Rehabilitación. Medidas sanitarias.

\begin{abstract}
One of the most daunting challenges faced by people with visual disability in their daily lives is travel. Safe and independent travel calls not only for perceptive, conceptual and orientation skills,

Bellón, M.J., Blocona, C., y Matey, M.Á. (2021). La autonomía de las personas con discapacidad visual y los programas de rehabilitación en tiempos de pandemia. RED Visual: Revista Especializada en Discapacidad Visual, 77, 100-108. https://doi.org/10.53094/TVOH6953.
\end{abstract}


but also for a safe and intuitive environment, with readily interpretable clues for decision-making. This article addresses the health and safety measures implemented in the wake of the pandemic that have added to the obstacles hindering this community's orientation and mobility. In times of visual supremacy, designing solutions to such problems is neither a straightforward nor a realistic endeavour, for the results do not always depend on personal skills. Rather, any regulation or change adopted in the surrounds should be broached from the perspective of how information is perceived by people with no or highly deficient vision. The article also discusses the changes taking place in the way ONCE rehabilitation services are being delivered during the pandemic.

\section{Key words}

Independent travel. Safe distancing. Rehabilitation. Health measures.

\section{Introducción}

La cara más dura de la pandemia por covid-19 ha sido la enfermedad masiva, la pérdida de vidas y la difícil situación económica, pero más allá de esas repercusiones directas, también ha condicionado nuestros comportamientos y ha forzado un cambio en la forma de relacionarnos, comunicarnos, entretenernos, trabajar y estudiar. Sin embargo, aunque el virus constituye una amenaza real para todos, las consecuencias han tenido un efecto desigual en las personas en función de su edad, género, actividad habitual, lugar de residencia y otros factores.

Las personas con discapacidad han sido especialmente vulnerables y aquellas que padecen una pérdida grave de visión no han sido una excepción. Según el informe de 2020 Potenciar las voces: nuestras vidas, nuestra decisión (Unión Mundial de Ciegos [UMC], 2020), elaborado por la UMC a partir de casi 900 encuestas realizadas en todo el mundo, son muchos los retos que han tenido que superar, siendo los más complejos aquellos que tienen relación directa con Transporte y movilidad; Independencia, autonomía y dignidad, y Salud mental y bienestar.

La Organización Nacional de Ciegos Españoles (ONCE) ha proporcionado a todos sus afiliados el soporte preciso para garantizar el acceso a los productos básicos, a la tecnología, al apoyo psicosocial y el bienestar a través de sus profesionales especializados y del voluntariado. Sin embargo, son muchos los obstáculos que tienen que superar para mantener su autonomía y vida independiente en la situación actual.

Bellón, M.J., Blocona, C., y Matey, M.Á. (2021). La autonomía de las personas con discapacidad visual y los programas de rehabilitación en tiempos de pandemia. RED Visual: Revista Especializada en Discapacidad Visual, 77, 100-108. https://doi.org/10.53094/TVOH6953. 
Así mismo, desde la ONCE se ha realizado un estudio sobre el colectivo de afiliados en relación al impacto que ha tenido el coronavirus, atendiendo a la preocupación de los mismos, cómo se han sentido durante este periodo y la actuación de la Institución y sus servicios sociales, resultados que se exponen en este número de la revista. ${ }^{1}$

\section{Afectaciones de la pandemia en la autonomía de las personas con discapacidad visual}

Ser autónomo implica ser capaz de desarrollar uno mismo las actividades básicas de la vida diaria. Aunque la discapacidad visual reduce las habilidades necesarias para desplazarse, realizar las tareas domésticas, acceder a información, etc., muchas personas con problemas graves de visión logran niveles de autonomía muy altos gracias a la sustitución sensorial (oído, tacto, cinestesia...), el aprendizaje de técnicas específicas, el uso de materiales facilitadores, las adaptaciones del entorno y la ayuda de otras personas.

La incorporación de las medidas sanitarias para evitar la propagación del virus, las modificaciones en el entorno físico y las nuevas pautas de relación social están constituyendo auténticos desafíos para mantener un nivel aceptable de autonomía por los motivos que analizamos a continuación.

\subsection{Incorporación de las medidas higiénicas}

El uso de mascarillas, la higiene máxima y el distanciamiento social son medidas que no representan, por sí mismas, inconveniente alguno para las personas con discapacidad visual, que pueden aplicarlas de forma eficiente en sus entornos privados. Sin embargo, tienen algunas consecuencias que están influyendo negativamente en su funcionamiento.

- Uso de mascarillas: algunos estudios indican que pueden implicar sensación de pérdida de capacidad auditiva, porque el sonido se percibe distorsionado. Pero no solo se ha agravado el problema de quién escucha, sino también del que habla, que tiene que elevar el tono de voz para hacerse entender. El oído es un

1 Ver, en este mismo número, el artículo firmado por Víctor Omar Dabbagh Rollán, pp. 13-42 (N. del E.).

Bellón, M.J., Blocona, C., y Matey, M.Á. (2021). La autonomía de las personas con discapacidad visual y los programas de rehabilitación en tiempos de pandemia. RED Visual: Revista Especializada en Discapacidad Visual, 77, 100-108. https://doi.org/10.53094/TVOH6953. 
sentido compensatorio fundamental para quienes no cuentan con el apoyo del lenguaje gestual ni con la posibilidad de observar el contexto para favorecer la interpretación, problemática aún más grave cuando se padece sordoceguera.

- Gel hidroalcohólico: está en nuestro día a día, en las tiendas, restaurantes, puestos de trabajo, transportes e incluso en plena calle. Pero su ubicación no está estandarizada, por lo que resulta muy difícil su localización, y existe, además, una variedad de mecanismos para su dispensación (palanca, pedal, sensor...) cuya manipulación es compleja y poco intuitiva.

- «No tocar»: pulsar botoneras, manejar teclados y sujetarse en los asideros del transporte son acciones muy frecuentes, que adquieren aún más protagonismo cuando el tacto es la única vía perceptiva para identificar productos, detectar referencias, leer carteles en braille, etc.

\subsection{Mantenimiento de la distancia de seguridad}

La recomendación de mantener la distancia física de seguridad, ya sea en estático o en movimiento, requiere visualizar perfectamente el espacio para verificar el número de personas concentradas, anticipar sus movimientos y determinar la posición y el lugar más adecuados para ubicarse.

Las personas con discapacidad visual requieren, en muchas situaciones cotidianas, solicitar ayuda o informaciones, tanto al público en general como al personal de los comercios e instituciones. El uso de esta estrategia sin sobrepasar la distancia recomendada no resulta viable, ya que muchas veces se requiere, además, el contacto físico para el acompañamiento y guiado.

\subsection{Compras y gestiones}

La atención telefónica y telemática han sustituido, en muchos casos, a la presencial para realizar tanto compras como trámites y gestiones. Aunque entre el colectivo son muchos los que tienen acceso a la tecnología, la brecha digital está muy presente en una parte considerable, que, de ninguna manera, tiene esta posibilidad. Algunas cadenas comerciales han instaurado la figura del «asistente personal» para prestar apoyo a aquellos que puedan precisarlo, pero todavía es una iniciativa que no está, ni mucho menos, generalizada.

Bellón, M.J., Blocona, C., y Matey, M.Á. (2021). La autonomía de las personas con discapacidad visual y los programas de rehabilitación en tiempos de pandemia. RED Visual: Revista Especializada en Discapacidad Visual, 77, 100-108. https://doi.org/10.53094/TVOH6953. 


\subsection{El desplazamiento}

Sin duda, el desplazamiento ha sido una de las actividades más damnificadas, y que, por ser esencial para realizar cualquier acción, está limitando las posibilidades de autonomía.

- Las colas de espera en las puertas de comercios y locales han sido habituales desde los primeros días del confinamiento. La localización del final de la fila y el movimiento al ritmo necesario, manteniendo la distancia física, son acciones casi imposibles si no se pueden controlar visualmente.

- Indicaciones e instrucciones: los espacios tienen limitaciones de aforo y han impuesto otras reglas - como el uso de guantes- de las que se informa mediante notas en caracteres visuales ubicadas en accesos, pasillos, salas de espera, ascensores y mostradores. Entre las personas con discapacidad visual, solo las que tienen resto visual pueden obtener la información de dichos carteles, pero acercarse para leerlos no siempre es factible, porque su ubicación suele ser inaccesible.

- Circuitos de circulación: para evitar la proximidad de personas que deambulan por los espacios públicos, se han definido direcciones obligatorias, a través de líneas y flechas dibujadas en el suelo que solo pueden interpretarse si pueden verse. Además de este inconveniente, estos circuitos se han convertido en «lugares desconocidos» para muchos que antes los recorrían con eficiencia, porque su «mapa cognitivo» del espacio se ha visto modificado y ya no pueden utilizar las referencias memorizadas que les permitían desplazarse por los puntos de interés.

- Cambios en el entorno urbano: las actividades se han reducido y también los sonidos. La información sonora del tráfico ya no es una referencia fiable para orientarse o realizar un cruce. Además, muchas calles son ahora peatonales, han proliferado las terrazas y nuevos elementos de mobiliario urbano (zonas de descanso y de juego) que ocupan, a veces, el itinerario peatonal, constituyendo verdaderos obstáculos y contribuyendo a la desorientación. Se ha sustituido el vehículo y el transporte por la bicicleta, el patinete y otros vehículos de movilidad personal que no siempre circulan de forma ordenada y lo hacen por cualquier punto de la vía pública, representando un verdadero peligro para los peatones.

Bellón, M.J., Blocona, C., y Matey, M.Á. (2021). La autonomía de las personas con discapacidad visual y los programas de rehabilitación en tiempos de pandemia. RED Visual: Revista Especializada en Discapacidad Visual, 77, 100-108. https://doi.org/10.53094/TVOH6953. 
- En el transporte resulta aún más difícil mantener la distancia y, además, se ha reducido considerablemente la atención personal al usuario por parte de los conductores, que realizan su trabajo aislados del resto del público mediante mamparas.

Como respuesta a estas necesidades y dificultades específicas, desde la Dirección de Autonomía Personal, Accesibilidad, Tecnología e Innovación de la ONCE se elaboró la Guía de recomendaciones sobre seguridad y distanciamiento social para personas afiliadas (Organización Nacional de Ciegos Españoles, 2020), que mereció la mención del Gobierno de España como ejemplo de protección frente a la pandemia para las personas con discapacidad visual grave.

Este documento, además de recomendar el cumplimiento de los protocolos sanitarios generales, analiza los ámbitos de la vida cotidiana donde el seguimiento de los mismos entraña dificultades, proponiendo además algunas estrategias atenuantes.

Pero, a pesar de todos los esfuerzos, las barreras en la movilidad y el transporte han aumentado la dependencia, sobre todo en quienes no tienen un puesto de trabajo, no cursan estudios reglados o no tienen la obligación de desplazarse para atender a sus familiares. Son personas que anteriormente dedicaban su tiempo a la práctica de relaciones sociales y a las actividades de ocio y tiempo libre, actualmente muy limitadas por el cierre de los espacios públicos donde se prestan.

\section{La rehabilitación en los tiempos de la covid-19}

El Servicio de Rehabilitación es uno de los que la ONCE ofrece a sus afiliados. Su finalidad es la consecución de la autonomía personal, proporcionando técnicas, estrategias y recursos para realizar las actividades cotidianas. El equipo está compuesto por tres perfiles profesionales: oftalmólogo, óptico y técnico de rehabilitación.

Tras la suspensión de casi tres meses en la prestación del servicio durante la etapa del confinamiento, a mediados del mes de junio se retomaron los programas. Además de incorporar los protocolos de seguridad en el trabajo, comunes a todos los centros de la ONCE y a sus trabajadores, la proximidad física que implica la metodología en rehabilitación determinó que, desde la Dirección de Autonomía Personal, Accesibilidad, Tecnología e Innovación, se elaborara la Guía de recomendaciones técnicas para el Servicio de Rehabilitación.

Bellón, M.J., Blocona, C., y Matey, M.Á. (2021). La autonomía de las personas con discapacidad visual y los programas de rehabilitación en tiempos de pandemia. RED Visual: Revista Especializada en Discapacidad Visual, 77, 100-108. https://doi.org/10.53094/TVOH6953. 
Articulada en cuatro bloques, la guía está dirigida a gestores y profesionales, y recoge, en primer lugar, una serie de recomendaciones generales para la prestación de todos los servicios de la ONCE, relacionadas con la obligatoriedad de la cita previa, la reorganización de las salas de espera, la utilización de los equipos de protección personal, la presencia de un único acompañante y el procedimiento a seguir para la desinfección, tanto de los espacios como de los materiales. El resto de bloques proporcionan pautas específicas para el entrenamiento en Actividades de la vida diaria, Orientación y Movilidad, y Optimización del funcionamiento visual.

Actualmente, el Servicio de Rehabilitación está funcionando con relativa normalidad, pero se han detectado ciertas divergencias respecto a las intervenciones que se realizaban con anterioridad al estado de alarma que conviene destacar.

En primer lugar, la incertidumbre y el miedo son determinantes para que muchos de los nuevos afiliados solo quieran recibir atención para resolver aspectos muy puntuales, posponiendo la consecución de objetivos significativos hasta que mejore la situación.

Así mismo, los cierres perimetrales establecidos para contener la covid-19, fijados tanto a nivel municipal como comarcal, han obstaculizado la asistencia a los servicios de rehabilitación para recibir la atención. Si bien es cierto que los profesionales se desplazan a los entornos de residencia de las personas afiliadas, algunas intervenciones, como las evaluaciones oftalmológica y óptica, y ciertos entrenamientos solo pueden llevarse a cabo en las sedes de la ONCE.

Algunas personas ya rehabilitadas han solicitado un programa para reforzar la seguridad y eficiencia en sus desplazamientos, destrezas que se han deteriorado a causa de la inactividad durante el confinamiento. Es previsible que este tipo de demandas vaya en aumento, a medida que se supere el escenario actual y se pueda ir recobrando la normalidad cotidiana.

Sin duda, otro factor que está influyendo en el funcionamiento habitual del servicio es la disminución significativa de actividades presenciales de animación sociocultural en los centros, que, para muchas personas, constituían una motivación para desplazarse y el eje en el que basan su autonomía para realizar otras muchas tareas.

En definitiva, aún es pronto para analizar la repercusión cualitativa que la inesperada pandemia ha tenido en el área de la rehabilitación, pero es evidente que la atención se

Bellón, M.J., Blocona, C., y Matey, M.Á. (2021). La autonomía de las personas con discapacidad visual y los programas de rehabilitación en tiempos de pandemia. RED Visual: Revista Especializada en Discapacidad Visual, 77, 100-108. https://doi.org/10.53094/TVOH6953. 
ha visto alterada. Por este motivo, será necesario anticiparse a situaciones análogas que pudieran producirse en el futuro, analizando, por ejemplo, las posibilidades que podría tener la «telerrehabilitación». Es evidente que la metodología de trabajo en esta área y el uso de estrategias personalizadas imponen una reflexión exhaustiva sobre las ventajas e inconvenientes de la atención telemática, limitando estrictamente los objetivos que podrían cubrirse con este método y el perfil del usuario que podría beneficiarse.

\section{Conclusiones}

Las medidas sanitarias tuvieron que tomarse improvisadamente y adaptándose al curso de los acontecimientos con el fin de minimizar los riesgos. Al adoptarlas, no se han tenido en cuenta las necesidades de las personas con discapacidad visual, y su imperativa puesta en práctica ha abocado a muchas de ellas al aislamiento, la inactividad y la dependencia, viéndose limitada su capacidad de controlar, afrontar y tomar, por propia iniciativa, decisiones personales acerca de cómo vivir de acuerdo con las normas y preferencias (Ley 39/2006, de 14 de diciembre, de Promoción de la Autonomía Personal y Atención a las personas en situación de dependencia [PDF]).

Sin duda, la sociedad ya no será la misma una vez superada la emergencia sanitaria. Es esperable que en el futuro se resuelvan las disfunciones que hemos planteado en estas líneas, y que los responsables tengan en cuenta la exigencia de adoptar medidas adaptadas a todos, garantizando el acceso a la información y un desarrollo urbano más inclusivo. Por su parte, instituciones y profesionales tenemos el reto de encontrar alternativas que garanticen el derecho a la autonomía y la prestación normalizada de los servicios. Y una vez más, queda para las personas afectadas el derecho a que se escuchen sus voces y el desafío de superarse a sí mismas para ser independientes.

\section{Referencias bibliográficas}

Organización Nacional de Ciegos Españoles (2020). Guía de recomendaciones sobre seguridad y distanciamiento social para personas afiliadas. Organización Nacional de Ciegos Españoles. https://club.once.es/afiliados/pensando-en-ti/Informacion-accesibley-recursos-utiles-sobre-el-COVID-19/recomendaciones-de-seguridad-y-distanciamientosocial-para-personas-afiliadas-a-la-once.

Bellón, M. J., Blocona, C., y Matey, M.Á. (2021). La autonomía de las personas con discapacidad visual y los programas de rehabilitación en tiempos de pandemia. RED Visual: Revista Especializada en Discapacidad Visual, 77, 100-108. https://doi.org/10.53094/TVOH6953. 
Unión Mundial de Ciegos (2020). Potenciar las voces: nuestras vidas, nuestra decisión Resumen ejecutivo [Word]. Unión Mundial de Ciegos.

M. Jesús Bellón Fernández. Técnica de Rehabilitación. Asesora de la Dirección de Autonomía Personal, Accesibilidad, Tecnología e Innovación. Dirección General de la ONCE. Calle del Prado, 24; 28014 Madrid (España). Correo electrónico: mjbf@once.es.

Concepción Blocona Santos. Técnica de Rehabilitación. Asesora de la Dirección de Autonomía Personal, Accesibilidad, Tecnología e Innovación. Dirección General de la ONCE. Calle del Prado, 24; 28014 Madrid (España). Correo electrónico: cbs@once.es.

M. a Ángeles Matey García. Técnica de Rehabilitación. Asesora de la Dirección de Autonomía Personal, Accesibilidad, Tecnología e Innovación. Dirección General de la ONCE. Calle del Prado, 24; 28014 Madrid (España). Correo electrónico: manm@once.es. 